\title{
FDG-PET/CT in infections: the imaging method of choice?
}

\author{
Andor W. J. M. Glaudemans • Alberto Signore
}

Published online: 11 August 2010

(C) The Author(s) 2010. This article is published with open access at Springerlink.com

\author{
"Caelum, non animum, mutant qui trans mare \\ currunt" \\ They change the sky, not their soul, who run across \\ the sea. \\ Horace, Epistles 1.11.27
}

In the wake of the United States' regulations on the use of ${ }^{18} \mathrm{~F}$-fluorodeoxyglucose (FDG), the Spanish medicines regulatory agency last year stated that the use of FDG with PET, although well established in the areas of cancer, cardiac medicine and neurology, is not yet sufficiently established, within the European Union, in the diagnosis of infectious and inflammatory diseases, and therefore should not be authorised as a diagnostic radiopharmaceutical in these conditions. This position was contested by other EU member states and the dispute was eventually referred to the European Medicines Agency (EMEA). The EMEA Committee for Medicinal Products for Human Use (CHMP)

\footnotetext{
A. W. J. M. Glaudemans • A. Signore

Department of Nuclear Medicine and Molecular Imaging, University Medical Centre Groningen, University of Groningen, Groningen, The Netherlands
}

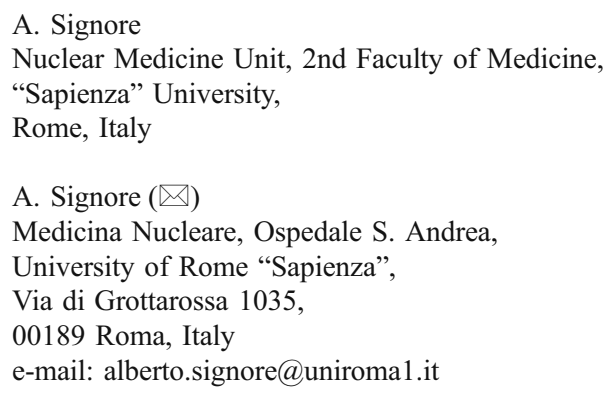

concluded that the benefits of FDG outweigh its risks, and that its use should, therefore, be authorised across the EU.

The CHMP deemed adequately documented a series of indications for the use of FDG in infectious or inflammatory diseases, namely:

1. Localisation of abnormal foci to guide the aetiological diagnosis in the presence of fever of unknown origin (FUO)

2. Diagnosis of infection in: suspected chronic infection of bone and/or adjacent structures (osteomyelitis, spondylitis, discitis or osteitis including presence of metallic implants), diabetes with suspicion of Charcot's neuroarthropathy, osteomyelitis and/or soft tissue infection, painful hip prosthesis, vascular prosthesis, and fever in AIDS

3. Detection of the extent of inflammation in: sarcoidosis, inflammatory bowel disease, and vasculitis involving the great vessels

4. Therapeutic follow-up of unresectable alveolar echinococcosis, in which it may be used in the search for active localisations of the parasite during medical treatment and after treatment discontinuation

However, although the use of FDG in infections has EMEA approval, this approach still lacks the support of solid, evidence-based criteria. In certain situations, it cannot yet be considered an adequate replacement for labelled white blood cell (WBC) imaging or anti-granulocyte monoclonal antibody $(\mathrm{MoAb})$ imaging. In this paper, we focus specifically on those infectious and inflammatory diseases in which questions remain over the use of FDG-PET as a tool for diagnostic evaluation and treatment follow-up. 


\section{Fever of unknown origin}

For more than 20 years labelled leukocyte imaging using ${ }^{99 \mathrm{~m}} \mathrm{Tc}-\mathrm{HMPAO}$ or ${ }^{111} \mathrm{In}$-oxine has been the radionuclide procedure of choice for diagnosing most infections, due to the ability of radiolabelled granulocytes to migrate to the foci of infection. Even years ago, high sensitivity and specificity values were reported for this technique in patients with FUO or a focal infection, while a negative study virtually excludes an infection. Furthermore, if there is a high suspicion of an infection, based on an increased leukocyte count and on an elevated erythrocyte sedimentation rate and C-reactive protein level, this procedure remains the diagnostic technique of choice, thus limiting the use of ${ }^{18} \mathrm{~F}$-FDG-PET/CT to cases of FUO with low probability of infection. However, it is worth pointing out that, to date, there exist no clearly established criteria for determining probability of infection, and a consensus statement would therefore be required.

FDG is a radiopharmaceutical that accumulates in infections, malignancies and inflammatory diseases. This non-specificity is extremely valuable in FUO, which is caused, primarily, by these three conditions. The disadvantage of FDG's low specificity (lower than that of labelled WBCs) is that it cannot always discriminate between infection and neoplastic disease. Other disadvantages are the considerable costs of FDG-PET and the fact that PET facilities are still scarce in many developing countries [1].

Several recent studies, retrospective and prospective, have drawn attention to the added value of FDG-PET over conventional techniques, recorded in $40-70 \%$ of patients with FUO $[2,3]$. Most studies also stress the technique's high negative predictive value in the assessment of FUO.

A recent study analysed the value of FDG-PET/CT in children with FUO or unexplained signs of inflammation without fever and found the technique to be a valuable diagnostic tool in these subjects in whom a non-traumatic method of depicting inflammation in the whole body is obviously particularly useful [4]. The value of FDG-PET/CT was also evaluated in critically ill, mechanically ventilated patients suspected of having an infection or inflammatory process. Comparisons of scans with clinical follow-up data yielded overall accuracy of $91 \%$ and, on this basis, the authors stated that FDG-PET/CT scanning is of additional value in critically ill patients. A normal FDG-PET/CT scan ruled out an infection requiring prolonged antibiotic therapy or drainage [5]. In patients with human immunodeficiency virus (HIV) and FUO, FDG-PET/CT emerged as a valuable tool and was helpful for diagnosis, especially when CT anatomical landmarks were added to PET findings [6].

All these studies stress the advantages of FDG-PET/CT for evaluating patients with FUO. However, the studies performed in this clinical setting in recent years are weakened by the use of different definitions of FUO and by the lack of a structured diagnostic protocol, which has resulted in varying efficacy data [3].

For the time being, the probability of infection remains the most useful criterion for choosing between FDG-PET/ $\mathrm{CT}$ and WBC imaging. In the presence of low and medium probability FDG is the more indicated technique, whereas WBC imaging should be performed when the probability of infection is high. However, the body of evidence on the utility of FDG-PET/CT in patients with FUO is growing and this technique is probably destined to become the preferred diagnostic procedure in the future, especially when a definite diagnosis cannot easily be achieved.

\section{Bone infections: osteomyelitis and spondylodiscitis}

For acute osteomyelitis, WBC imaging combined-if necessary - with bone marrow imaging or MoAb imaging, is very reliable, with overall accuracy of approximately $90 \%$ [7]. FDG-PET/CT, used in combination with conventional methods, may have limited value in the diagnosis of uncomplicated cases of acute osteomyelitis; conversely, it may play an important role in patients with chronic osteomyelitis, particularly those with previously documented osteomyelitis and suspected recurrence, or presenting with symptoms of osteomyelitis for more than 6 weeks. FDG-PET/CT can also be used to monitor response to antimicrobial treatment and to develop criteria for deciding when treatment can safely be stopped [3].

The performance of WBC imaging is poor in spondylitis and spondylodiscitis: up to $50 \%$ of all patients with spondylodiscitis show photopenic lesions due to encapsulation of the infection, and therefore relatively hampered migration of leukocytes; as a result the specificity of the method is reduced [8]. In these patients, FDG-PET/CT gave much better results: sensitivities ranging from $94 \%$ to $100 \%$ and specificities ranging from $87 \%$ to $100 \%$. In a recent retrospective study, FDG-PET/CT had a strong impact on the clinical management (initiation or prolongation of antibiotic therapy or recourse to surgical intervention) of $52 \%$ of patients with infectious spondylitis [9]. A recent review article highlights the clinical role of FDG-PET/CT in diagnosing spinal infections, especially in patients with contraindications to MRI, and in evaluation of the postoperative spine [10].

In summary, the use of FDG-PET/CT is clearly indicated in spondylodiscitis (even though there is a need for clearer criteria for positivity and for clarification of the role of the standard uptake volume), whereas $\mathrm{WBC}$ and $\mathrm{MoAb}$ imaging remain the gold standard imaging techniques in patients with suspected osteomyelitis in peripheral bones. 


\section{Diabetic foot infection}

The role of WBC imaging in the diagnosis of diabetic pedal osteomyelitis has been extensively investigated, disclosing sensitivities-using ${ }^{111}$ In-oxine-ranging from $72 \%$ to $100 \%$ and specificities ranging from $67 \%$ to $100 \%$. With this approach, poor spatial resolution and lack of bony landmarks makes the differentiation of soft tissue from bone infection difficult; instead, much better results are achieved using SPECT/CT [7]. FDG-PET/CT was found to be highly sensitive in excluding osteomyelitis in the diabetic foot, and to usefully complement MRI, particularly in cases with positive MRI findings. Conventional imaging like MRI or bone scanning, also lacks specificity as a means of distinguishing osteomyelitis in the diabetic foot from Charcot's neuroarthropathy. Preliminary data suggest that FDG-PET/CT could play a role in assessing complicated and uncomplicated diabetic osteoarthropathy, being able to provide accurate assessment of patients with metal implants who may not be suitable candidates for MRI, and to correctly distinguish osteomyelitis from neuroarthropathy [3]. In a recent prospective study conducted in 110 patients with complicated diabetic foot, FDG-PET/CT was found to be a highly specific imaging modality for the diagnosis of osteomyelitis and was deemed a useful complementary imaging modality for use with MRI [11].

However, our group recently investigated the role of FDG-PET/CT in the diabetic foot and found-even with sequential imaging-low overall diagnostic accuracy. These findings suggest that FDG-PET/CT cannot yet replace WBC imaging, particularly when $\mathrm{WBC}$ is acquired using SPECT/CT modality and bone marrow imaging is added for Charcot's foot.

\section{Hip and knee prosthesis infections}

WBC imaging - if necessary combined with bone marrow imaging - is currently the radionuclide imaging procedure of choice for diagnosing prosthetic joint infections, on account of its almost 90\% accuracy [12]. MoAb imaging also shows very high accuracy, thus limiting the need for alternative modalities. Theoretically, FDG-PET/CT has the potential to detect infection in hip and, to a lesser extent, knee prostheses. It is not affected by artefacts due to metal implants and it provides images with higher resolution than those produced using conventional nuclear medicine techniques. However, non-infectious reactions around the neck of the prosthesis are common months and even years after surgery, and these may influence the diagnosis. Increased FDG uptake around the neck and/or head should not be interpreted as a finding suggestive of infection. Studies in the past that compared WBC imaging with FDG-PET scanning in prosthetic joint infections showed better results with WBC imaging, which proved more sensitive and more specific than FDG-PET. The lack of specificity of the FDGPET/CT modality prompted definition of interpretation criteria [8]. To date, $\mathrm{WBC} / \mathrm{MoAb}$ imaging remains the gold standard technique for evaluating prosthetic infections and the question of whether FDG-PET/CT can provide satisfactory diagnostic accuracy in these patients is still debated. If well-designed prospective studies prove able to establish criteria for infection and aseptic loosening, it is possible that there may, in the future, be a role for FDG$\mathrm{PET} / \mathrm{CT}$ in the evaluation of prosthetic joint infections.

\section{Vascular graft infections}

Vascular graft infections are uncommon but severe complications that can occur long after surgery. Sensitivities of WBC imaging for diagnosing graft infections range from $53 \%$ to $100 \%$, and specificities from $50 \%$ to $100 \%$. Falsepositive results are often reported and have been associated with perigraft haematomas, thrombosed grafts, bleeding and recent surgery. There is little literature regarding the role of FDG-PET/CT in the assessment of vascular graft infections, but that which is available suggests that this modality is a reliable tool in this clinical setting and also indicates that $\mathrm{CT}$ is mandatory for the correct localisation of FDG accumulation [13]. Indeed it is not uncommon for FDG-PET/CT to detect infection of vascular grafts even when the angio-CT results are negative; in the past, comparison of the two modalities showed FDG-PET to be superior to CT scanning alone. Combining FDG-PET with $\mathrm{CT}$ increases the test specificity and therefore the diagnostic accuracy because the precise anatomical localisation of increased FDG uptake, thanks to PET/CT, allows accurate differentiation between graft and adjacent soft tissue infection, leading to more accurate diagnosis and thus an optimal therapeutic strategy. This added value of PET/CT over PET alone has been described by several investigators. In particular, Spacek et al., prospectively evaluating FDG$\mathrm{PET} / \mathrm{CT}$ in 76 patients with a total of 96 vascular prosthetic grafts, found the technique to be reliable, having an accuracy of $>95 \%$ in $75 \%$ of the prostheses (the ones with intense uptake) [14]. These authors' interesting attempt to identify interpretation criteria in order to increase the diagnostic accuracy of FDG-PET/CT is an endeavour that others would do well to emulate. Again it emerges that interpretation criteria (qualitative versus quantitative) are mandatory and require multi-centre validation.

In general, it is agreed that FDG-PET/CT is the modality of choice in vascular graft prosthesis. Menezes et al. examined the question of the ideal imaging time in 17 patients with atherosclerotic abdominal aorta aneurysms 
and found no significant differences between imaging at $1 \mathrm{~h}$ and $3 \mathrm{~h}$ after injection [15]. Similar findings have been recorded in osteomyelitis and FUO.

\section{Vasculitis}

As regards the use of FDG-PET/CT in the evaluation of large-vessel vasculitis, a few brief comments suffice. The modality has proven validity in this setting, with sensitivity values ranging from $77 \%$ to $92 \%$ and specificities ranging from $89 \%$ to $100 \%$. FDG-PET/CT has proven utility in the initial diagnosis of patients suspected of having vasculitis, particularly those who present with non-specific symptoms, in the identification of areas of increased FDG uptake requiring biopsy, and in the evaluation of the extent of disease [16].

\section{Evaluation of therapy in infectious or inflammatory diseases}

The CHMP report mentions the use of FDG-PET/CT in treatment evaluation only in connection with unresectable alveolar echinococcosis. Despite this lack of attention, it is our view that FDG-PET/CT could play a pivotal role in therapy assessment. The evolution of FDG uptake reflects the efficacy of the medical treatment, and its careful assessment can lead to better modulation of the drug dosage or prompt a radical modification of the therapeutic strategy. It has been shown to be a valuable imaging tool for assessing treatment efficacy in systemic sarcoidosis, large-vessel arteritis, tuberculosis and aspergillosis [17]. In vasculitis, FDG-PET/CT also has proven utility in assessing response to therapy [16]. From this perspective, it is possible to conceive of a role for FDG-PET/CT in monitoring therapy response and early relapses of inflammatory bowel diseases, even though only limited data are currently available in this regard [18]. As regards the monitoring of antibiotic therapy in osteomyelitis, there is, as yet, no published evidence to support this indication.

\section{AIDS and tuberculosis}

We have already discussed the role of FDG-PET/CT in patients with HIV and FUO [6]. However, FDG-PET also plays a major role in the assessment and management of HIV-1-infected patients [3]. FDG-PET data have shown that HIV-1 infection progresses by distinct anatomical steps, with involvement of the upper torso preceding involvement of the lower part of the body, and that the degree of FDG uptake is related to viral load [19]. FDG uptake by the lymph nodes of HIV-positive patients was found to be inversely related to CD4 count, thereby supporting the theory of CD4 cell depletion through forced lymph node homing [20]. In the clinical setting, FDG-PET has been shown to allow the differentiation of AIDS-related opportunistic infections and malignancies, and to allow monitoring of the side effects of highly active antiretroviral treatment [21]. Comparison of FDG uptake in patients who had received antiretroviral therapy (ART) and patients who were ART-naïve revealed different patterns of FDG uptake: all the ART-treated patients with either suppressed or high viraemia showed a normal pattern, while the ART-naïve patients with high viraemia displayed multiple foci of increased glucose metabolism in the lymph nodes. Together, this finding and the finding of a correlation between the well-established markers of progression to AIDS and positive FDG-PET in ART-naïve patients seem to confer prognostic value on FDG uptake [22].

Data on FDG-PET imaging in patients suffering from tuberculosis (TB) are very limited. Pulmonary TB commonly causes an increase in FDG uptake, whereas uptake is low in tuberculous pleural effusion. Sites of extrapulmonary TB can be detected with FDG-PET, including disease involving the central nervous system and joint and bone TB [23].

In general, it is difficult in patients with high FDG uptake to differentiate between a malignancy, HIV infection and TB. Several authors have shown that FDG uptake continues to increase over time in malignant lesions, whereas in inflammatory lesions uptake decreases or remains stable. However, studies conducted to assess the potential impact of double-phase FDG-PET versus routine staging in patients suffering from TB confirmed that it is extremely difficult to distinguish TB from malignant involvement [24]. This was also borne out by the findings of a study in HIV-infected children, in whom FDG-PET scanning proved unable to discriminate reliably between malignant and inflammatory pathology [25].

However, differentiation between malignancies, AIDS and TB is extremely important. In patients with HIV and $\mathrm{TB}$, early identification and diagnosis are the keys to effective control of the disease. Correct identification of TB is crucially important in order to start anti-TB treatment and delay antiretroviral treatment for HIV. The presentation of TB in the HIV-infected patient is different from that observed in the HIV-negative patient: apical predominance is less pronounced, while consolidation, cavitations and haematogenic disseminations are less prevalent. In this context, FDG-PET/CT may aid in the diagnosis.

Aggressive lymphoid proliferations should be differentiated from generalised lymphadenopathy, which does not have a negative outcome, and here again FDG-PET may play a central role. FDG-PET scanning could also be 
indicated for monitoring the efficacy of TB treatment, while FDG activity may also be a useful tool for evaluating and excluding sites of active disease in the context of targeted screening for latent TB infection before immunosuppressive treatment.

Finally, a new strategy for the application of FDG-PET/ $\mathrm{CT}$ in AIDS patients needs to be put in place and would certainly prove beneficial in the management of opportunistic infections (TB), facilitating diagnosis and improving response to therapy.

\section{Conclusions and future perspectives}

Over the past decade, FDG-PET/CT has emerged as a rapidly evolving diagnostic tool in infectious diseases. Its clinical impact on diagnosis, staging and evaluation of therapy, already high, is destined to increase in the future. However, FDG-PET/CT is not always the best radionuclide imaging technique to choose in the presence of infectious diseases. The value of $\mathrm{WBC} / \mathrm{MoAb}$ scanning in different infectious diseases has been well established over the past 20 years and should not be overlooked. Larger prospective studies evaluating the role of FDG-PET/CT in the clinical management of patients with infectious diseases are needed, and in many cases it remains to be seen whether this modality will prove able to record the high sensitivity and specificity values already shown by WBC/MoAb imaging.

In many inflammatory conditions there is also a pressing need to develop clear criteria for interpreting FDG-PET/CT.

In an attempt to combine the best of both worlds, leukocytes were labelled with ${ }^{18} \mathrm{~F}$-FDG. Although promising preliminary results were obtained, FDG was found to be rapidly released from the labelled leukocytes. Further major drawbacks were the labelling time, the short half-life of the tracer, the costs, and the need for specific expertise [1]. Yet this is far from the end of the road for leukocyte PET/CT imaging: the common clinical need for specific and anatomically precise detection of infection, the availability of the components necessary for performing leukocyte $\mathrm{PET} / \mathrm{CT}$, and their lack of toxicity and adverse effects are all factors making it worthwhile to go on exploring the scope for PET/CT imaging, in larger prospective studies [26]. In this context, the ongoing quest to find better labelling agents may well result in the identification of the ideal PET/CT radiopharmaceutical for imaging infection and inflammation.

"Caelum, non animum, mutant qui trans mare currunt": while we may change technique and/or radiopharmaceutical, we nevertheless remain steadfast in our determination to achieve diagnostically accurate imaging of infection.
Open Access This article is distributed under the terms of the Creative Commons Attribution Noncommercial License which permits any noncommercial use, distribution, and reproduction in any medium, provided the original author(s) and source are credited.

\section{References}

1. Signore A, Soroa VA, De Vries EF. Radiobelled white blood cells or FDG for imaging on inflammation and infection? Q J Nucl Med Mol Imaging. 2009;53(1):23-5.

2. Balink H, Collins J, Bruyn G, Gemmel F. F-18 FDG PET/CT in the diagnosis of fever of unknown origin. Clin Nucl Med. 2009;34 (12):862-8.

3. Basu S, Chryssikos T, Moghadam-Kia S, Zhuang H, Torigian DA, Alavi A. Positron emission tomography as a diagnostic tool in infection: present role and future possibilities. Semin Nucl Med. 2009;39(1):36-51.

4. Jasper N, Däbritz J, Frosch M, Loeffler M, Weckesser M, Foell D. Diagnostic value of [(18)F]-FDG PET/CT in children with fever of unknown origin or unexplained signs of inflammation. Eur $\mathrm{J}$ Nucl Med Mol Imaging. 2010;37(1):136-45.

5. Simons KS, Pickkers P, Bleeker-Rovers CP, Oyen WJ, van der Hoeven JG. F-18-fluorodeoxyglucose positron emission tomography combined with CT in critically ill patients with suspected infection. Intensive Care Med. 2010;36(3):504-11.

6. Castaigne C, Tondeur M, de Wit S, Hildebrand M, Clumeck N, Dusart M. Clinical value of FDG-PET/CT for the diagnosis of human immunodeficiency virus-associated fever of unknown origin: a retrospective study. Nucl Med Commun. 2009;30 (1):41-7.

7. Palestro CJ, Love C, Bhargava KK. Labeled leukocyte imaging: current status and future directions. Q J Nucl Med Mol Imaging. 2009;53(1):105-23.

8. van der Bruggen W, Bleeker-Rovers CP, Boerman OC, Gotthardt M, Oyen WJ. PET and SPECT in osteomyelitis and prosthetic bone and joint infections: a systematic review. Semin Nucl Med. 2010;40 (1):3-15.

9. Ito K, Kubota K, Morooka M, Hasuo K, Kuroki H, Mimori A. Clinical impact of $18 \mathrm{~F}-\mathrm{FDG}$ PET/CT on the management and diagnosis of infectious spondylitis. Nucl Med Commun. 2010;31 (8):691-8.

10. Gemmel F, Rijk PC, Collins JM, Parlevliet T, Stumpe KD, Palestro CJ. Expanding role of $18 \mathrm{~F}$-fluoro-D-deoxyglucose PET and PET/CT in spinal infections. Eur Spine J. 2010;19 (4):540-51.

11. Nawaz A, Torigian DA, Siegelman ES, Basu S, Chryssikos T, Alavi A. Diagnostic performance of FDG-PET, MRI, and plain film radiography (PFR) for the diagnosis of osteomyelitis in the diabetic foot. Mol Imaging Biol. 2010;12(3):335-42.

12. Love C, Marwin SE, Palestro CJ. Nuclear medicine and the infected joint replacement. Semin Nucl Med. 2009;39(1):66-78.

13. Keidar Z, Nitecki S. FDG-PET for the detection of infected vascular grafts. Q J Nucl Med Mol Imaging. 2009;53(1):35-40.

14. Spacek M, Belohlavek O, Votrubova J, Sebesta P, Stadler P. Diagnostics of "non-acute" vascular prosthesis infection using 18F-FDG PET/CT: our experience with 96 prostheses. Eur J Nucl Med Mol Imaging. 2009;36(5):850-8.

15. Menezes LJ, Kotze CW, Hutton BF, Endozo R, Dickson JC, Cullum I, et al. Vascular inflammation imaging with $18 \mathrm{~F}-\mathrm{FDG}$ PET/CT: when to image? J Nucl Med. 2009;50(6):854-7.

16. Zerizer I, Tan K, Khan S, Barwick T, Marzola MC, Rubello D, et al. Role of FDG-PET and PET/CT in the diagnosis and management of vasculitis. Eur J Radiol. 2010;73(3):504-9. 
17. Imperiale A, Federici L, Lefebvre N, Braun JJ, Pfumio F, Kessler R, et al. F-18 FDG PET/CT as a valuable imaging tool for assessing treatment efficacy in inflammatory and infectious diseases. Clin Nucl Med. 2010;35:86-90.

18. Glaudemans AW, Maccioni F, Mansi L, Dierckx RA, Signore A. Imaging of cell trafficking in Crohn's disease. J Cell Physiol. 2010;223(3):562-71.

19. Sathekge M, Goethals I, Maes A, Van de Wiele C. Positron emission tomography in patients suffering from HIV-1 infection. Eur J Nucl Med Mol Imaging. 2009;36(7):1176-84.

20. Sathekge M, Maes A, Kgomo M, Van de Wiele C. Fluorodeoxyglucose uptake by lymph nodes of HIV patients is inversely related to CD4 cell count. Nucl Med Commun. 2010;31(2):137-40.

21. Sathekge M, Goethals I, Maes A, Van de Wiele C. Positron emission tomography in patients suffering from HIV-1 infection. Eur J Nucl Med Mol Imaging. 2009;36(7):1176-84.
22. Lucignani G, Orunesu E, Cesari M, Marzo K, Pacei M, Bechi G, et al. FDG-PET imaging in HIV-infected subjects: relation with therapy and immunovirological variables. Eur J Nucl Med Mol Imaging. 2009;36(4):640-7.

23. Sathekge M, Maes A, Kgomo M, Stoltz A, Pottel H, Van de Wiele C. Impact of FDG PET on the management of TBC treatment. A pilot study. Nuklearmedizin. 2010;49(1):35-40.

24. Hadley GP, Naude F. Malignant solid tumour, HIV infection and tuberculosis in children: an unholy triad. Pediatr Surg Int. 2009;25 (8):697-701.

25. Sathekge M, Maes A, Al Nahhas A, Rubello D, Chiti A. What impact can fluorine-18 fluorodeoxyglucose PET/computed tomography have on HIV/AIDS and tuberculosis pandemic? Nucl Med Commun. 2009;30(4):255-7.

26. Dumarey N. Imaging with FDG labeled leukocytes: is it clinically useful? Q J Nucl Med Mol Imaging. 2009;53(1):89-94. 